\title{
Improvement of pregnancy rate after deep uterine artificial insemination with frozen-thawed cauda epididymal spermatozoa in Hanwoo cattle
}

\author{
Sung-Sik Kang, Ui-Hyung Kim, Jun Sang Ahn, Jeong Il Won and Sang-Rae Cho* \\ Hanwoo Research Institute, National Institute Animal Science, Rural Development Administration, Pyeongchang 25340, \\ Korea
}

Received May 10, 2021

Revised May 18, 2021

Accepted May 18, 2021

*Correspondence

Sang-Rae Cho

E-mail: chosr@korea.kr

ORCID

https://orcid.org/0000-0003-0209-6248

\begin{abstract}
In the present study, we examined if deep uterine artificial insemination (DUAI) can improve the pregnancy rate of artificial insemination (AI) using epididymal spermatozoa (ES) in Hanwoo cattle. The estrus cycles of 88 Hanwoo cows were synchronized, and 17 cows were artificially inseminated using the DUAI method with ES, 20 cows were artificially inseminated via the uterine body (BUAI) method with ES, and as a control, 51 cows were inseminated by using the BUAl method with ejaculated spermatozoa from 1 proven bull after frozen thawing. The pregnancy rate of the DUAI method (58.8\%) was higher than that of the BUAI method (25.0\%, $p=0.0498$ ). The motility of ES was examined immediately after thawing and after 3 and $6 \mathrm{~h}$ of incubation. The rapid progressive sperm motility of the control group was significantly higher than that of the ES group immediately after thawing and after 3 and $6 \mathrm{~h}$ of incubation $(p<0.05)$. The straight line velocity and average path velocity of the ES group after $6 \mathrm{~h}$ of incubation were significantly lower than those in the control group $(p<0.05)$. The linearity and amplitude of lateral head of ES were lower than those at $6 \mathrm{~h}(p<0.05)$. The flagellar beat cross frequency and hyperactivation of ES were lower than the control spermatozoa immediately after thawing and at $3 \mathrm{~h}(p<0.05)$. These motility parameters suggested that ES had a low motility and fertilization ability compared to the control spermatozoa. After frozen-thawing and $3 \mathrm{~h}$ of incubation, the percentage of live spermatozoa with intact acrosomes in the ES was significantly lower than that in ejaculated spermatozoa $(p<0.05)$. Our findings suggested that the DUAI method can overcome the low pregnancy rate of ES, despite the low motility, viability, and fertilization ability of ES.
\end{abstract}

Keywords: artificial insemination, epididymal spermatozoa, Hanwoo, motility, pregnancy rate

\section{INTRODUCTION}

Artificial insemination (AI) is a powerful assisted reproduction technology used in the dairy and beef cattle industries. Commonly, ejaculated semen is collected from bulls using the artificial vagina method (Vishwanath, 2003); however this method is not feasible for bulls with poor libido or injury. Therefore, the collection of epididymal sperm is recommended as an alternative and rescue method for valuable male gametes (Cary et al., 2004). The 
fertilization ability of epididymal sperm and the embryo developmental competency after fertilization in vitro have been demonstrated for bulls (Martins et al., 2007) and felines (Kunkitti et al.,2016). The fertilization ability of epididymal spermatozoa by AI has also been demonstrated in several species, including boars (Okazaki et al., 2012), bulls (Kozdrowski et al., 2011), dogs (Hori et al., 2004), and felines (Tsutsui et al., 2003). The calving rate after AI with epididymal spermatozoa was reportedly $22.2 \%$ in European bison (Kozdrowski et al., 2011) and 38.9\% in spotted buffalo (Yulnawati et al., 2013). However, previous reports have focused only on AI using frozen-thawed epididymal spermatozoa to verify the fertility of epididymal spermatozoa, irrespective of their low motility.

Frozen-thawed semen is commonly introduced into the uterine body and passes through the cervix of the female. Semen introduced in the uterine body will move to the ampullary-isthmic junction, and a low number of motile epididymal spermatozoa will reach the ampullary-isthmic junction compared to ejaculated spermatozoa (Hunter, 2003). This low motility of epididymal spermatozoa decreases the possibility of oocyte encounter and results in a low conception rate after AI. Methods that improve the typically low pregnancy rates of AI with epididymal spermatozoa would be of great economic importance. The deep uterine horn AI (DUAI) method, with frozen-thawed semen, is one such recommended method (Verberckmoes et al., 2004) and has been shown to improve the pregnancy rate of cows by $10 \%$ to $20 \%$ compared to that of the uterine body AI (BUAI) method (López-Gatius and Camón-Urgel, 1988; Senger et al., 1988).

Here, we hypothesized that the DUAI method with frozen-thawed epididymal spermatozoa can improve the pregnancy rate of Hanwoo cows. We examined the efficacy of the DUAI method with epididymal spermatozoa by comparing the pregnancy rate of Hanwoo cows after using the DUAI or BUAI methods with frozen-thawed epididymal spermatozoa, and the BUAI method with frozenthawed ejaculated spermatozoa from one proven bull (control) (Experiment 1). We also examined the motility of epididymal and ejaculated spermatozoa following thawing $(0 \mathrm{~h})$ and incubation (3 and $6 \mathrm{~h}$ ) (Experiment 2), and examined the viability and acrosome integrity of epididymal spermatozoa after frozen-thawing (Experiment 3).

\section{MATERIALS AND METHODS}

\section{Castration, recovery of epididymal spermatozoa, and cryopreservation}

Two testicles with epididymides were castrated from eight Hanwoo bulls (means \pm standard deviations; age, $457.5 \pm 2.1$ days; scrotal circumference, $31.5 \pm 0.7 \mathrm{~cm}$; body weight, $386.5 \pm 6.4 \mathrm{~kg}$ ). Castrations were conducted from November 2016 to December 2018 at the Hanwoo Research Institute, NIAS, Pyeongchang, Republic of Korea. For general anesthesia, $1.0 \mathrm{~mL}$ per $100 \mathrm{~kg}$ of xylazine hydrochloride (Rompun, Bayer, Korea) was injected into the jugular vein. After 5-15 min of injection, the body, legs, and head were fixed with a rope. The scrotal circumference was estimated with a scrotal measuring tape, and the skin of the testis was incised using a scalpel. The testes with epididymides were recovered, and the surgical site was sutured. Twenty milliliters of dexamethasone disodium phosphate (Dexolone-20, Samyang Anifarm, Korea) and $20 \mathrm{~mL}$ of antibiotics (G.C.GPS-INJ., Green Cross Veterinary, Korea) were intramuscularly injected. The extracted testes with epididymides were delivered to the laboratory within $1 \mathrm{~h}$. In the laboratory, the tunica albuginea of the testicles was removed, each epididymis with vas deferens was isolated from, and the blood vessels and debris tissue on the surface of the cauda epididymis were removed. Epididymal spermatozoa were recovered by flushing or mincing (Kang et al., 2018). Semen freezing was performed as described previously (Kang et al., 2018) with slight modification. Briefly, spermatozoa were diluted to $40-50 \times 10^{6}$ cells $/ \mathrm{mL}$ with semen freezing extender and preserved at $4^{\circ} \mathrm{C}$ for $4 \mathrm{~h}$. Semen dilution was loaded into a $0.5 \mathrm{~mL}$ straw, kept at $3 \mathrm{~cm}$ above liquid nitrogen $\left(\mathrm{LN}_{2}\right)$ for $14 \mathrm{~min}$, and then plunged into $\mathrm{LN}_{2}$.

\section{Artificial insemination and pregnancy diagnosis}

For DUAI with epididymal spermatozoa, frozen epididymal semen from two bulls were used for the AI of 17 cows (8 and 9 cows per bull). For BUAI with epididymal spermatozoa, frozen thawed epididymal semen from two bulls was used for the AI of 20 cows ( 10 cows per bull). For the control, frozen ejaculated semen from one proven Hanwoo bull was used for the AI (i.e., BUAI) of 51 cows. To synchronize the estrus cycle of the Hanwoo cows, an intravaginal progesterone insert (EAZI-BREED CIDR 1900, Zoetis, Belgium) was inserted into the vagina of each cow 
for 7 days. After 7 days, the intravaginal progesterone insert was removed, and $5 \mathrm{~mL}$ of prostaglandin F $2 \alpha$ (Lutalyse, Zoetis, Belgium) was injected intramuscularly. After 2.5 days, AI was conducted and each cow received an intramuscular injection of $2 \mathrm{~mL}$ gonadotrophin-releasing hormone (GnRH; Fertagyl, Inervet International, Germany). A second AI was conducted the following morning. For the DUAI method, a deep uterine AI device (YT-gun, Yamato Tech, Co., Ltd., Japan) was used. After introduction of the DUAI device into the uterine body, a flexible catheter was extended $\sim 15 \mathrm{~cm}$ to the deep uterus, and frozen-thawed semen was introduced into the horn of the deep uterus. For the BUAI method, a conventional cassou AI gun (No. 17025/000, Minitube, Germany) was used, and frozenthawed semen was introduced into the body of the uterus. After 80 to 90 days of AI, conception was examined by ultrasound (My Lab 30 VET Gold, Esaote SpA, Italy) and rectal palpation by a well-trained technician.

\section{Measurement of spermatozoa motility}

Spermatozoa motility was measured based on previously described methods (Kang et al., 2018). In brief, frozen straws were thawed in water at $37^{\circ} \mathrm{C}$ for $40 \mathrm{~s}$, mixed in a $1.5 \mathrm{~mL}$ microtube, and incubated at $38.5^{\circ} \mathrm{C}$ in $5 \% \mathrm{CO}_{2}$ in air. Sperm motility was evaluated after thawing $(0 \mathrm{~h})$ and after incubation ( 3 and $6 \mathrm{~h}$ ) using a computer-assisted sperm analysis system (Sperm Class Analyzer, MicroOptic, Spain). Semen $(3 \mu \mathrm{L})$ was placed onto four-chamber slides with a depth of $20 \mu \mathrm{m}$ from the bottom slide to the upper cover (Art. No. SC-20-01-04-B, Leja, Nieuw-Vennep, Netherlands). At least 1,000 spermatozoa in 4-5 fields in a chamber were classified as motile or dead. The motility and motility parameters were evaluated, as follows: straight line velocity (VSL, $\mu \mathrm{m} / \mathrm{s}$ ), curvilinear velocity (VCL, $\mu \mathrm{m} / \mathrm{s})$, average path velocity (VAP, $\mu \mathrm{m} / \mathrm{s})$, linearity (LIN = VSL/VCL $\times 100, \%)$, straightness (STR, \%), flagellar beat cross frequency $(\mathrm{BCF}, \mathrm{Hz})$, and amplitude of lateral head (ALH, $\mu \mathrm{m})$.

\section{Evaluation of viability and acrosome integrity of spermatozoa}

The viability and acrosome integrity of spermatozoa were evaluated as previously described (Kovács and Foote, 1992) with slight modification. In brief, $20 \mu \mathrm{L}$ of semen was mixed with $20 \mu \mathrm{L}$ of trypan blue solution and smeared on glass slides. After air drying, slides were immersed in a fixative solution for $5 \mathrm{~min}$, air dried, and then stained with $7.5 \%$ Giemsa solution for $10-12 \mathrm{~h}$. A minimum of 200 spermatozoa were counted under a microscope ( $\times 400$ magnification) and divided into 7 groups: live spermatozoa with intact acrosomes (LIA), live spermatozoa with damaged acrosomes (LDA), dead spermatozoa with intact acrosomes (DIA), dead spermatozoa with damaged acrosomes (DDA), LIA with distal cytoplasmic droplets, DIA with distal cytoplasmic droplets, DDA with distal cytoplasmic droplets.

\section{Experimental design}

In experiment 1 , frozen-thawed epididymal spermatozoa (from two bulls) were introduced to the deep uterine horn to examine the influence of DUAI on the pregnancy rate compared to the BUAI method with epididymal spermatozoa (from two bulls) and with frozen-thawed ejaculated spermatozoa (from one proven bull) i.e., the control. In experiment 2 , the motility of frozen-thawed epididymal spermatozoa was evaluated after thawing (0 h) and after incubation ( 3 and $6 \mathrm{~h}$ ) by CASA. As a control, frozen-thawed ejaculated spermatozoa were used (from one proven bull). In experiment 3 , the viability and acrosome integrity of epididymal spermatozoa were evaluated using frozen-thawed epididymal spermatozoa (from two bulls) after frozen-thawing $(0 \mathrm{~h})$ and after incubation $(3 \mathrm{~h})$.

\section{Statistical analysis}

The number of pregnant cows in the DUAI, BUAI, and control groups were compared using Fisher's exact test. The motility percentage, rapid progressive sperm motility, motility parameters, viability, and acrosome integrity (LIA, LDA, DIA, DDA) of the epididymal spermatozoa group and control spermatozoa group were compared using a one-way ANOVA followed by a post-hoc analysis (Duncan Multiple Range Test). All analyses were performed using SAS (Statistical Analysis System software, version 9.4).

\section{RESULTS}

\section{Experiment 1}

The pregnancy rate after the DUAI method (58.8\%) was higher than that of the control group (56.9\%) and significantly higher after the BUAI method $(25.0 \%, p=0.048)$ (Table 1). 
Table 1. The pregnancy rate of Hanwoo cows after deep uterine artificial insemination (DUAl) with epididymal spermatozoa and uterine body artificial insemination (BUAl) with epididymal and ejaculated spermatozoa (control)

\begin{tabular}{ccccc}
\hline Semen deposition method & Origin of semen & No. cows inseminated & No. cows pregnant & Pregnancy rate (\%) \\
\hline DUAl & Epididymis & 17 & 10 & $58.8^{*}$ \\
BUAl & Epididymis & 20 & 5 & 25.0 \\
BUAl & Ejaculated & 51 & 29 & 56.9 \\
\hline
\end{tabular}

*Asterisk indicates a significantly higher value compared to BUAl with epididymis group $(p=0.498)$. Semen from one bull was used as the control and frozen-thawed semen was introduced into the body of the uterus via BUAl.
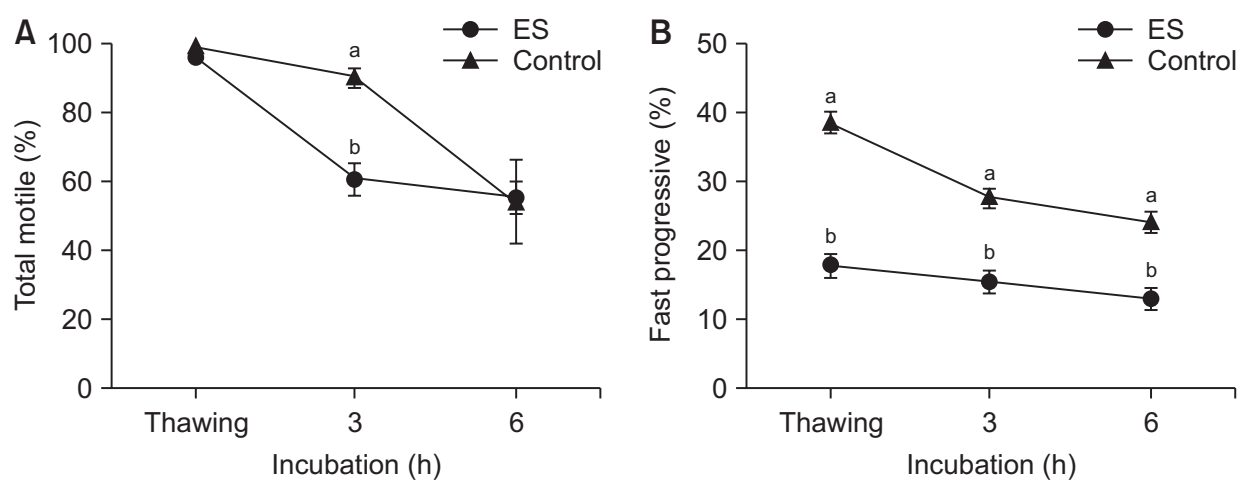

Fig. 1. Spermatozoa motility of frozen-thawed epididymal spermatozoa at thawing $(0 \mathrm{~h})$ and following incubation periods of $3 \mathrm{~h}$ and $6 \mathrm{~h}$ after thawing. (A) Total motile (\%), (B) Fast progressive (\%). ES, epididymal sperm; Control, ejaculated sperm. ${ }^{a, b}$ values with different letters are significantly different within the same incubation period $(p<0.05)$.

\section{Experiment 2}

The total motility of the epididymal spermatozoa group was lower than that of the control spermatozoa group after 3 h of incubation ( $p<0.05$, Fig. 1A), even though the motility of the epididymal spermatozoa was not significantly different after thawing $(0 \mathrm{~h})$ and $6 \mathrm{~h}$ of incubation $(p>0.05)$. The rapid progressive sperm motility percentage was higher in the control group than in the epididymal spermatozoa group immediately after thawing as well as after 3 and $6 \mathrm{~h}$ of incubation ( $p<0.05$, Fig. 1B). The spermatozoa motility parameters are shown in Fig. 2. The VCL of spermatozoa was significantly higher in the control group than in the epididymal spermatozoa group after 3 h of incubation ( $p<0.05$, Fig. $2 A)$. The VSL of spermatozoa was significantly greater in the control group than in the epididymal spermatozoa group at 3 and $6 \mathrm{~h}$ of incubation (Fig. 2B). The VAP of spermatozoa was significantly higher in the control group than in the epididymal spermatozoa group at 3 and $6 \mathrm{~h}$ of incubation $(p<0.05$, Fig. 2C). The LIN of spermatozoa was significantly higher in the control group than in the epididymal spermatozoa group after $6 \mathrm{~h}$ of incubation (Fig. 2D). The STR of spermatozoa was significantly greater in the control group than in the epididymal spermatozoa group (Fig. 2E). The
ALH of spermatozoa was significantly greater in the control group than in the epididymal spermatozoa group after $6 \mathrm{~h}$ of incubation (Fig. 2F). The BCF of spermatozoa was significantly greater in the control group than in the epididymal spermatozoa group immediately after thawing and after $3 \mathrm{~h}$ of incubation (Fig. 2G). The hyperactivity of the spermatozoa was significantly greater in the control group than in the epididymal group after thawing and after $3 \mathrm{~h}$ of incubation (Fig. $2 \mathrm{H}$ ).

\section{Experiment 3}

The percentage of LIA was significantly higher in ejaculated sperm than in epididymal sperm after frozenthawing ( $p<0.05$, Table 2). The percentages of DDA, LIA with distal cytoplasmic droplets, and DIA with distal cytoplasmic droplets were significantly higher in epididymal sperm than in ejaculated sperm after thawing $(p<0.05)$. After $3 \mathrm{~h}$ of incubation, the percentage of LIA was significantly higher in ejaculated sperm than in epididymal sperm $(p<0.05)$. After 3 h of incubation, the percentage of LIA with distal cytoplasmic droplets and DIA with cytoplasmic droplets were significantly higher in ejaculated sperm than in epididymal sperm $(p<0.05)$. 

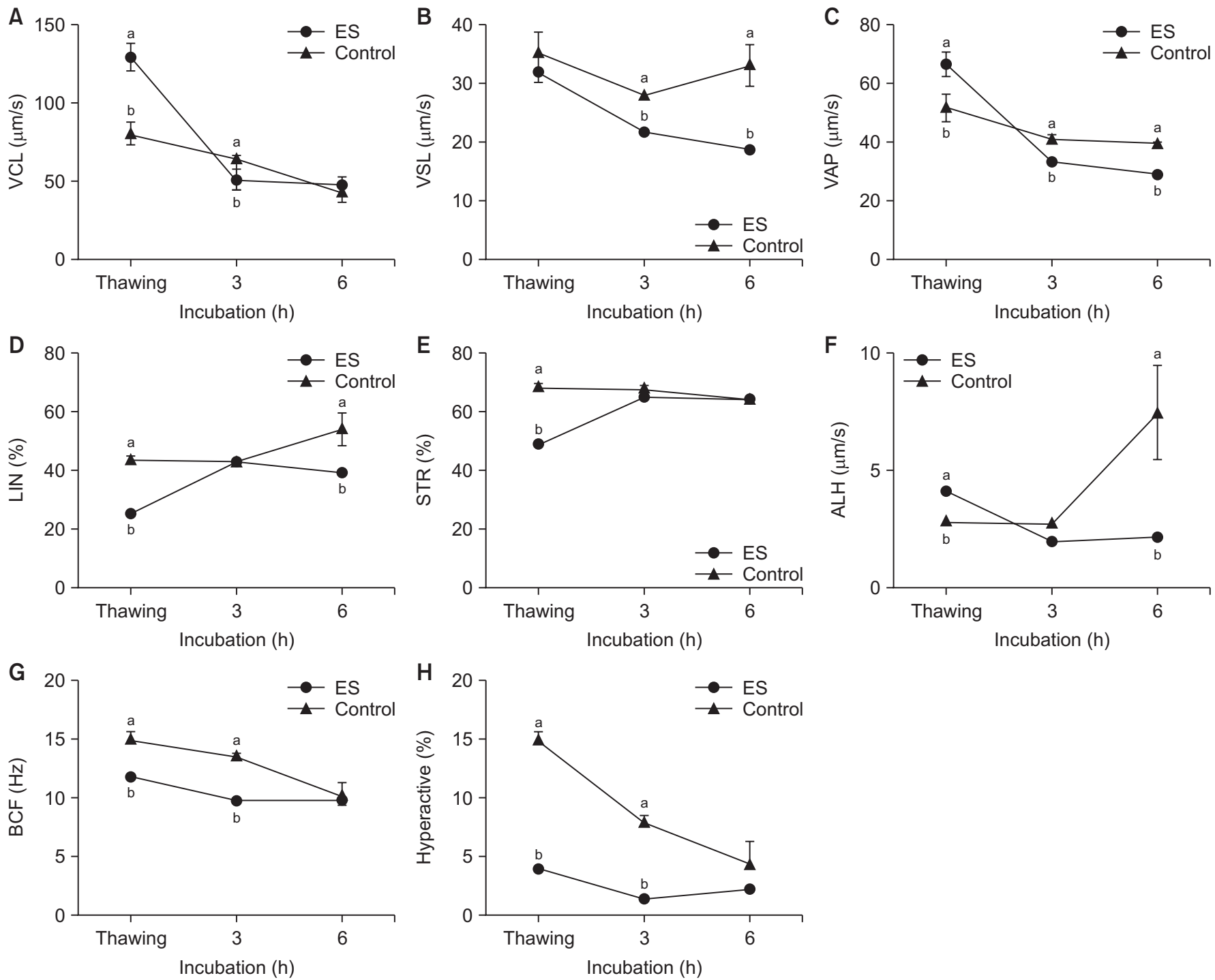

Fig. 2. Spermatozoa motility parameters of frozen-thawed epididymal spermatozoa at thawing $(0 \mathrm{~h})$ and following incubation periods of $3 \mathrm{~h}$ and $6 \mathrm{~h}$ after thawing. ES, epididymal sperm; Control, ejaculated sperm. (A) mean curvilinear velocity (VCL), (B) mean straight-line velocity (VSL), (C) mean average path velocity (VAP), (D) mean linearity (LIN = VSL/VCL [curvilinear velocity] $\times 100),($ E) mean straightness (STR), (F) mean amplitude of lateral head (ALH), (G) mean flagellar beat cross frequency (BCF), (H) mean spermatozoa hyperactivity. ${ }^{a, b}$ values with different letters are significantly different within the same incubation period $(p<0.05)$.

Table 2. The viability and acrosome integrity of epididymal spermatozoa from Hanwoo bulls after frozen-thawing

\begin{tabular}{|c|c|c|c|c|c|c|c|c|c|}
\hline \multirow{2}{*}{ Incubation (h) } & \multirow{2}{*}{ Origin of semen } & \multirow{2}{*}{ LIA } & \multirow{2}{*}{ LDA } & \multirow{2}{*}{ DIA } & \multirow{2}{*}{ DDA } & \multicolumn{4}{|c|}{ Distal cytoplasmic droplet with } \\
\hline & & & & & & LIA & LDA & DIA & DDA \\
\hline \multirow[t]{2}{*}{0} & Epdidymis & $52.3 \pm 5.0^{\mathrm{a}}$ & $0.2 \pm 0.2$ & $17.0 \pm 2.5$ & $7.4 \pm 1.1^{\mathrm{a}}$ & $17.7 \pm 2.7^{\mathrm{a}}$ & $0 \pm 0$ & $4.3 \pm 1.1^{\mathrm{a}}$ & $0.7 \pm 0.2$ \\
\hline & Ejaculated & $70.7 \pm 2.5^{b}$ & $0.1 \pm 0.1$ & $25.8 \pm 2.1$ & $1.5 \pm 0.5^{b}$ & $1.9 \pm 0.2^{b}$ & $0 \pm 0$ & $0 \pm 0^{b}$ & $0 \pm 0$ \\
\hline \multirow[t]{2}{*}{3} & Epdidymis & $23.0 \pm 2.8^{\mathrm{a}}$ & $0 \pm 0$ & $41.1 \pm 2.3$ & $16.8 \pm 2.5$ & $12.1 \pm 1.7^{\mathrm{a}}$ & $0 \pm 0$ & $5.9 \pm 1.5^{\mathrm{a}}$ & $0.9 \pm 0.4$ \\
\hline & Ejaculated & $44.6 \pm 3.4^{b}$ & $0 \pm 0$ & $46.6 \pm 2.8$ & $8.7 \pm 1.8$ & $0 \pm 0^{b}$ & $0 \pm 0$ & $0 \pm 0^{b}$ & $0 \pm 0$ \\
\hline
\end{tabular}

Frozen-thawed epididymal spermatozoa from two bulls were used ( $n=5$ replicates per bull). Frozen-thawed ejaculated spermatozoa from one bull was used as the control. LIA, live spermatozoa with intact acrosomes; LDA, live spermatozoa with damaged acrosomes; DIA, dead spermatozoa with intact acrosomes; DDA, dead spermatozoa with damaged acrosomes. 


\section{DISCUSSION}

In the present study, we assessed the pregnancy rate of two AI methods (DUAI and BUAI) involving frozen-thawed epididymal spermatozoa and, thus, their potential to improve the pregnancy rate of Hanwoo cows artificially inseminated with epididymal spermatozoa. In addition, we assessed the motility of frozen-thawed epididymal spermatozoa immediately after thawing and after 3 and $6 \mathrm{~h}$ of incubation. The present study is the first report of pregnancy research involving the DUAI method using frozenthawed epididymal spermatozoa in Hanwoo cattle.

As shown in Table 1, the BUAI group with epididymal spermatozoa showed a lower pregnancy rate compared to that of the control group (BUAI group with ejaculated spermatozoa). The DUAI method with epididymal spermatozoa showed a similar conception rate compared to that of the control group (BUAI with ejaculated spermatozoa). However, the DUAI group had higher pregnancy and calving rates compared to the control group (BUAI with ejaculated spermatozoa) (Meirelles et al., 2012). Compared to the BUAI method, the DUAI method reduces semen loss (López-Gatius, 2000) and reduces the time taken to reach the isthmus reservoir to encounter ovulated oocytes in the oviduct (Hunter, 2003). Spermatozoa that have low motility, such as sex-sorted spermatozoa, were shown to have a low conception rate after BUAI compared to non-sorted spermatozoa after BUAI (Seidel and Schenk, 2008). However, DUAI with sex-sorted spermatozoa with low motility showed a $22 \%$ higher pregnancy rate compared to BUAI with sex-sorted spermatozoa (Chang et al., 2017). From the perspective of sperm motility, the total motility percentage observed at $3 \mathrm{~h}$ and the rapid progressive sperm motility of epididymal spermatozoa at thawing, and after 3 and 6 h of incubation was decreased compared to that of ejaculated spermatozoa (Fig. 1). Spermatozoa with low fertility after AI have lower total motility and progressive motility percentages (Morrell et al., 2017). Therefore, we speculated that BUAI with epididymal spermatozoa will yield a low fertilization rate, despite the motility of the epididymal spermatozoa being similar to that of ejaculated spermatozoa (Fig. 1A). When epididymal spermatozoa were deposited in the uterine body, the conception rate of epididymal spermatozoa decreased drastically, as shown in Table 1 . This result indicated that the distance that deposited spermatozoa were required to move in the body of the uterus to the isthmus reservoir may have been shorter for the epididymal spermatozoa than for the control spermatozoa. Thus, we anticipated that the DUAI method with epididymal spermatozoa could decrease the amount of time taken to reach the isthmus reservoir.

As shown in Fig. 2, the VCL, VAP, and LIN of the epididymal spermatozoa after 3 and $6 \mathrm{~h}$ of incubation were low compared to the ejaculated spermatozoa in the control BUAI group. This result indicated that frozen-thawed epididymal spermatozoa can move a short distance compared to ejaculated spermatozoa when introduced into the body of the uterus. Spermatozoa with increased LIN showed a higher pregnancy rate after AI compared to spermatozoa with low LIN in bovine (Morrell et al., 2017). In human spermatozoa, VCL, VAP, and LIN have been shown to be positively correlated with the fertilization rate in vitro and the pregnancy rate in vivo (Donnelly et al., 1998). Therefore, BUAI using spermatozoa with low VSL, VAP, and LIN traits will yield a lower conception rate. In addition, frozen-thawed epididymal spermatozoa with low LIN were reportedly not able to move the same distance as control group spermatozoa over the same time period (Kang et al., 2015); consequently, the conception rate would be decreased. However, the DUAI method with frozen-thawed epididymal spermatozoa, which had low VCL, VAP, and LIN traits, showed a similar conception rate compared to the BUAI method with frozenthawed ejaculated spermatozoa. This result indicated that the deep uterus deposition method can overcome the low conception rate of epididymal spermatozoa. As shown in Fig. 2E, the STR of epididymal spermatozoa after thawing was significantly lower than that of the control group spermatozoa. The low STR of spermatozoa indicates that epididymal spermatozoa have low fertilizability (Donnelly et al., 1998). As shown in Fig. 2F, the ALH of the control group spermatozoa at $6 \mathrm{~h}$ of incubation was higher than that of the epididymal spermatozoa $(p<0.05)$. As shown in Fig. 2G, the BCF of the control group spermatozoa immediately after thawing and after a $3 \mathrm{~h}$ incubation was significantly higher than that of the epididymal spermatozoa. We speculated that motile spermatozoa with high ALH and BCF are more likely to reach the fertilization site and show increased oocyte penetration (Chamberland et al., 2001). As shown in Fig. 2H, the control group spermatozoa showed high hyperactivation during thawing and after $3 \mathrm{~h}$ of incubation. Thus, the strong hyperactiva- 
tion of the control group spermatozoa suggested that the spermatozoa had sufficient energy to penetrate the cervical mucus (Aitken et al., 1985) and oocyte (Suarez and Dai, 1992; Stauss et al., 1995). Consequently, epididymal spermatozoa have low motility and a lower fertilization ability compared to ejaculated spermatozoa after frozenthawing.

From the perspective of spermatozoa acrosomal integrity, the percentage of live acrosome-reacted spermatozoa was significantly lower in the epididymal spermatozoa compared to the ejaculated spermatozoa at thawing and after $3 \mathrm{~h}$ of incubation (Table 2). Our results are consistent with those of a previous report that showed that an increased percentage of acrosome-reacted spermatozoa was positively correlated with a low conception rate in bovine (Whitfield and Parkinson, 1992). The cytoplasmic droplets of spermatozoa are composed of two types of droplets: proximal and distal cytoplasmic droplets. The proximal and distal cytoplasmic droplets at the spermatozoa tail were judged as abnormal and related to the reduction in fertility (Amann et al., 2000). Spermatozoa with proximal cytoplasmic droplets showed lower fertility compared to spermatozoa without cytoplasmic droplets in bovine (Amann et al., 2000). However, there is no conclusive evidence that spermatozoa with distal cytoplasmic droplets affect fertility in cattle. AI with boar spermatozoa revealed that spermatozoa with distal cytoplasmic droplets were negatively related to the pregnancy rate (Waberski et al., 1994). Therefore, we speculated that frozenthawed epididymal spermatozoa have reduced viability, normality of acrosomes, and fertility compared to frozenthawed ejaculated spermatozoa.

The DUAI device used in the present study was shown to increase the pregnancy rate after AI, irrespective of the low motility of epididymal spermatozoa. In addition, the use of a flexible catheter used might have another advantage in that it reduces the risk of uterine wall damage compared to the AI gun that is typically used for DUAI. Because the catheter could extend from the uterine body to the deep uterine horn without damaging the inner uterine wall, this insemination method may improve the pregnancy rate without negatively impacting the uterine wall (Verberckmoes et al., 2004). In contrast to AI using epididymal spermatozoa in vivo, the fertilization rate of epididymal spermatozoa in vitro was similar to that of ejaculated spermatozoa when epidydimal spermatozoa and oocytes were co-incubated, as shown in our previous report (Yang et al., 2015). We speculated that, under such conditions in vitro, they formed a small droplet or well. Epididymal spermatozoa in vitro do not consume energy moving to the isthmus of the uterus. Therefore, the in vitro fertilization rate of epididymal spermatozoa does not differ from that of ejaculated spermatozoa (Yang et al., 2015), despite epididymal spermatozoa showing low motility after incubation.

In the present study, we suggested that the DUAI method with low motility and low viability of epididymal spermatozoa can achieve similar pregnancy rate compared to the BUAI method with high motility and high viability of ejaculated spermatozoa. However, the limitations of this study are the small number of inseminated cows to prove efficacy of DUAI with low motility and low viability of epididymal spermatozoa. In addition, the pregnancy rate of DUAI with ejaculated spermatozoa was not examined, irrespective of ejaculated spermatozoa has high motility and high viability compared to epididymal spermatozoa. Therefore, in future study, we should examine the pregnancy rate using large number of inseminated cows with DUAI method by epididymal spermatozoa and ejaculated spermatozoa to verify the merit of DUAI with low quality of epididymal spermatozoa.

In conclusion, the DUAI method with epididymal spermatozoa can overcome the typically low pregnancy rate of $\mathrm{AI}$, irrespective of the low motility and low percentage of normal morphology of epididymal spermatozoa. In addition, this application method will greatly contribute to the production of offspring when genetically valuable animals show low fertility and conception abilities.

\section{ACKNOWLEDGEMENTS}

This study was conducted with the support of the Cooperative Research Program for Agriculture Science \& Technology Development project: "Development of improved technologies for preservation of Hanwoo bull semen and technology application”, PJ0143252021, RDA, Korea, and the 2021 Postdoctoral Fellowship Program of the Hanwoo Research Institute, NIAS, RDA, Korea. We thank MyungSuk Lee and Seok-Dong Lee at the Hanwoo Research Institute and Min-Hyung Jeon in the In-Woo Animal Hospital, Gochang, Korea for their assistance with epididymal spermatozoa recovery and freezing. 


\section{CONFLICTS OF INTEREST}

No potential conflict of interest relevant to this article was reported.

\section{AUTHOR CONTRIBUTIONS}

\author{
Conceptualization: Kang SS, Kim UH, Cho SR \\ Data curation: Kang SS, Ahn JS \\ Formal analysis: Kang SS, Won JI, Ahn JS \\ Funding acquisition: Cho SR, Kim UH \\ Investigation: Kang SS, Won JI, Ahn JS \\ Methodology: Kang SS \\ Project administration: Cho SR \\ Resources: Cho SR \\ Software: Kang SS, Won JI \\ Supervision: Cho SR
}

\section{AUTHOR'S POSITION AND ORCID NO.}

SS Kang, Postdoctoral Fellow,

https://orcid.org/0000-0002-9453-5377

UH Kim, Researcher,

https://orcid.org/0000-0002-2197-5080

JS Ahn, Postdoctoral Fellow,

https://orcid.org/0000-0001-7362-9270

JI Won Researcher,

https://orcid.org/0000-0003-3151-7144

SR Cho, Researcher,

https://orcid.org/0000-0003-0209-6248

\section{REFERENCES}

Aitken SC, Lippman ME, Kasid A, Schoenberg DR. 1985. Relationship between the expression of estrogen-regulated genes and estrogen-stimulated proliferation of MCF-7 mammary tumor cells. Cancer Res. 45:2608-2615.

Amann RP, Seidel GE Jr, Mortimer RG. 2000. Fertilizing potential in vitro of semen from young beef bulls containing a high or low percentage of sperm with a proximal droplet. Theriogenology 54:1499-1515.

Cary JA, Madill S, Farnsworth K, Hayna JT, Duoos L, Fahning ML. 2004. A comparison of electroejaculation and epididymal sperm collection techniques in stallions. Can. Vet. J. 45:35-41.

Chamberland A, Fournier V, Tardif S, Sirard MA, Sullivan R, Bailey JL. 2001. The effect of heparin on motility parameters and protein phosphorylation during bovine sperm capacita- tion. Theriogenology 55:823-835.

Chang LB, Chou CJ, Shiu JS, Tu PA, Gao SX, Peng SY, Wu SC. 2017. Artificial insemination of Holstein heifers with sexsorted semen during the hot season in a subtropical region. Trop. Anim. Health Prod. 49:1157-1162.

Donnelly ET, Lewis SE, McNally JA, Thompson W. 1998. In vitro fertilization and pregnancy rates: the influence of sperm motility and morphology on IVF outcome. Fertil. Steril. 70:305-314.

Hori T, Ichikawa M, Kawakami E, Tsutsui T. 2004. Artificial insemination of frozen epididymal sperm in beagle dogs. J. Vet. Med. Sci. 66:37-41.

Hunter RH. 2003. Advances in deep uterine insemination: a fruitful way forward to exploit new sperm technologies in cattle. Anim. Reprod. Sci. 79:157-170.

Kang SS, Kim UH, Jeon MH, Lee MS, Cho SR. 2018. Comparison of spermatozoa recovery methods on cauda epididymal sperm of Hanwoo bulls. J. Emb. Trans. 33:321-326.

Kang SS, Koyama K, Huang W, Yang Y, Yanagawa Y, Takahashi Y, Nagano M. 2015. Addition of D-penicillamine, hypotaurine, and epinephrine (PHE) mixture to IVF medium maintains motility and longevity of bovine sperm and enhances stable production of blastocysts in vitro. J. Reprod. Dev. 61:99-105.

Kovács A and Foote RH. 1992. Viability and acrosome staining of bull, boar and rabbit spermatozoa. Biotech. Histochem. 67:119-124.

Kozdrowski R, Niżański W, Dubiel A, Olech W. 2011. Possibilities of using the European bison (Bison bonasus) epididymal spermatozoa collected post-mortem for cryopreservation and artificial insemination: a pilot study. Reprod. Biol. Endocrinol. 9:31.

Kunkitti P, Axnér E, Bergqvist AS, Sjunnesson Y. 2016. In vitro fertilization using frozen-thawed feline epididymal spermatozoa from corpus and cauda regions. Theriogenology 86:1403-1408.

López-Gatius F. 2000. Site of semen deposition in cattle: a review. Theriogenology 53:1407-1414.

López-Gatius F and Camón-Urgel J. 1988. Increase of pregnancy rate in dairy cattle after preovulatory follicle palpation and deep cornual insemination. Theriogenology 29:10991103.

Martins CF, Rumpf R, Pereira DC, Dode MN. 2007. Cryopreservation of epididymal bovine spermatozoa from dead animals and its uses in vitro embryo production. Anim. Reprod. Sci. 101:326-331.

Meirelles C, Kozicki LE, Weiss RR, Segui MS, Souza A, dos Santos IW, Breda JCD. 2012. Comparison between deep intracornual artificial insemination (DIAI) and conventional artificial insemination (AI) using low concentration of spermatozoa in beef cattle. Braz. Arch. Biol. Technol. 55:371-374.

Morrell JM, Nongbua T, Valeanu S, Lima Verde I, LundstedtEnkel K, Edman A, Johannisson A. 2017. Sperm quality variables as indicators of bull fertility may be breed dependent. Anim. Reprod. Sci. 185:42-52.

Okazaki T, Akiyoshi T, Kan M, Mori M, Teshima H, Shimada M. 
2012. Artificial insemination with seminal plasma improves the reproductive performance of frozen-thawed boar epididymal spermatozoa. J. Androl. 33:990-998.

Seidel GE Jr and Schenk JL. 2008. Pregnancy rates in cattle with cryopreserved sexed sperm: effects of sperm numbers per inseminate and site of sperm deposition. Anim. Reprod. Sci. 105:129-138.

Senger PL, Becker WC, Davidge ST, Hillers JK, Reeves JJ. 1988. Influence of cornual insemination on conception in dairy cattle. J. Anim. Sci. 66:3010-3016.

Stauss CR, Votta TJ, Suarez SS. 1995. Sperm motility hyperactivation facilitates penetration of the hamster zona pellucida. Biol. Reprod. 53:1280-1285.

Suarez SS and Dai X. 1992. Hyperactivation enhances mouse sperm capacity for penetrating viscoelastic media. Biol. Reprod. 46:686-691.

Tsutsui T, Wada M, Anzai M, Hori T. 2003. Artificial insemination with frozen epididymal sperm in cats. J. Vet. Med. Sci. 65:397-399.

Verberckmoes S, Van Soom A, De Pauw I, Dewulf J, Vervaet C, de Kruif A. 2004. Assessment of a new utero-tubal junction insemination device in dairy cattle. Theriogenology 61:103115.

Vishwanath R. 2003. Artificial insemination: the state of the art. Theriogenology 59:571-584.

Waberski D, Meding S, Dirksen G, Weitze KF, Leiding C, Hahn R. 1994. Fertility of long-term-stored boar semen: influence of extender (Androhep and Kiev), storage time and plasma droplets in the semen. Anim. Reprod. Sci. 36:145-151.

Whitfield CH and Parkinson TJ. 1992. Relationship between fertility of bovine semen and in vitro induction of acrosome reactions by heparin. Theriogenology 38:11-20.

Yang BC, Kang SS, Park CS, Kim UH, Kim HC, Jeon GJ, Kim S, Lee SD, Lee HJ, Cho SR. 2015. Motility, fertilizability and subsequent embryonic development of frozen-thawed spermatozoa derived from epididymis in Hanwoo. J. Emb. Trans. 30:271-276.

Yulnawati Y, Maheshwari H, Rizal M, Boediono A. 2013. Frozen-thawed epididymal sperm quality and the success rate of artificial insemination in spotted buffaloes (Bubalus bubalis carabanensis). Buffalo Bull. 32(Special Issue 2):494497. 